Binghamton University

The Open Repository @ Binghamton (The ORB)

$9-2019$

\title{
Pairing electrostatic levitation with triboelectric transduction for high-performance self-powered MEMS sensors and actuators
}

\author{
Mark Pallay
}

Alwathiqbellah I. Ibrahim

Ronald N. Miles

Shahrzad Towfighian

Binghamton University--SUNY, stowfigh@binghamton.edu

Follow this and additional works at: https://orb.binghamton.edu/mechanical_fac

Part of the Mechanical Engineering Commons

\section{Recommended Citation}

M. Pallay, A. Ibrahim, R. Miles, S. Towfighian (2019) Pairing electrostatic levitation with triboelectric transduction for high-performance self-powered MEMS sensors and actuators, Applied Physics Letters, (2019)

This Article is brought to you for free and open access by the Mechanical Engineering at The Open Repository @ Binghamton (The ORB). It has been accepted for inclusion in Mechanical Engineering Faculty Scholarship by an authorized administrator of The Open Repository @ Binghamton (The ORB). For more information, please contact ORB@binghamton.edu. 


\title{
Pairing electrostatic levitation with triboelectric transduction for high-performance self-powered MEMS sensors and actuators
}

\author{
Mark Pallay, ${ }^{1, \text { a) }}$ Alwathiqbellah I Ibrahim, ${ }^{2, b)}$ Ronald N Miles, ${ }^{1, c)}$ and Shahrzad Towfighiann, d) \\ 1) Mechanical Engineering Department, Binghamton University \\ 4400 Vestal Parkway East \\ Binghamton, New York, 13901, USA \\ ${ }^{2)}$ Mechanical Engineering Department, University of Texas at Tyler \\ 3900 University Blvd. \\ Tyler, Texas, 75799, USA
}

(Dated: September 6, 2019)

\begin{abstract}
We demonstrate that an electrostatic levitation MEMS switch can be operated by applying mechanical pressure to a triboelectric generator. The toggling mechanism of the switch draws no current but requires a high actuating voltage, while the generator can supply a high voltage but only produces microwatts of power. The synergistic combination results in an entirely self-powered sensor and switch; the normally-closed MEMS switch can be toggled open by applying a threshold force to the generator without the need for any outside power or supplementary circuitry. A model of the MEMS switch and electrostatic force is validated with experimental data. An output voltage versus input force relationship for the generator is experimentally extracted.
\end{abstract}

Keywords: MEMS, Triboelectricty, Electrostatic Levitation, Sensors

Microelectromechanical systems (MEMS) that use electrostatic actuation are a staple of many commercial electronic products. Virtually all electrostatic actuators employ a parallel-plate electrode configuration to generate electrostatic forces that pull the two electrodes together. This configuration is widely used for electrostatic MEMS because it is extraordinarily reliable, robust, and well-suited to bulk fabrication ${ }^{1}$. However, this method of actuation has limitations that negatively affect the performance of the products that use them. Two of the primary limitations are the pull-in instability (collapse of two electrodes beyond certain applied voltage) and the short travel range of the movable electrode. The instability causes stiction, wear, dielectric charging, and breakdown ${ }^{2}$. Their short travel range limits their use to configurations that do not require significant electrode motion. Almost all electrostatic devices are designed to mitigate these problems, but that often limits performance.

Much effort has been made to create electrostatic MEMS devices that have alternative electrode configurations to the standard parallel-plate as a means of improving performance ${ }^{3-6}$. One method uses electrostatic levitation $^{3,7-12}$, which causes the two electrodes to be pulled away from each other rather than pulled together. A cross section of the electrode arrangement, first proposed in 2005 by $\mathrm{He}$ and Ben $\mathrm{Mrad}^{7}$, is shown in Figure 1 (a). In this scheme, three electrodes are fixed to a substrate and a cantilever is suspended above the cen-

\footnotetext{
a) mpallay1@binghamton.edu

b) aibrahim@uttyler.edu

c) miles@binghamton.edu

d)stowfigh@binghamton.edu; Address all correspondence to this author.
}

ter. The side electrodes are given a large voltage and the fixed center electrode receives a small bias voltage. The electric field produced from the side electrodes induces a net force upward because the field pulls on the top face of the beam more than the bottom. The bias voltage on the center electrode acts like a parallel-plate capacitor and pulls the beam back down.

This system can overcome the collapse caused by the pull-in instability between the beam and center electrode, which led to the creation of a reliable MEMS switch by the authors in their previous work ${ }^{13}$. This configuration also allows a much larger travel range because the beam can be pulled away from the substrate where there is nothing to impede its motion. This can boost signal-tonoise ratios in sensors and gives actuators a larger range of controllable motion. The major drawback of electrostatic levitation is the high voltage requirement between the center and side electrodes. It takes more than an order of magnitude higher voltage to generate an electrostatic levitation force that is comparable to a parallelplate electrode system.

One voltage generating mechanism that addresses the high voltage requirement of electrostatic levitation is triboelectricity ${ }^{14-20}$. Triboelectricity is contact electrification of materials and is responsible for generating most of the static charge that accumulates in everyday life. When two triboelectric materials contact each other, electrons move between them if one material has a high affinity to gain electrons and the other material has a high affinity to lose electrons. When the materials are separated from each other, they become equally and oppositely charged. The voltage generated can be on the order of thousands of volts. This, however, will produce only micro-amps of current, which makes powering sensing circuits difficult and raises their cost. Triboelectric generators are not coupled with parallel-plate electrostatic 

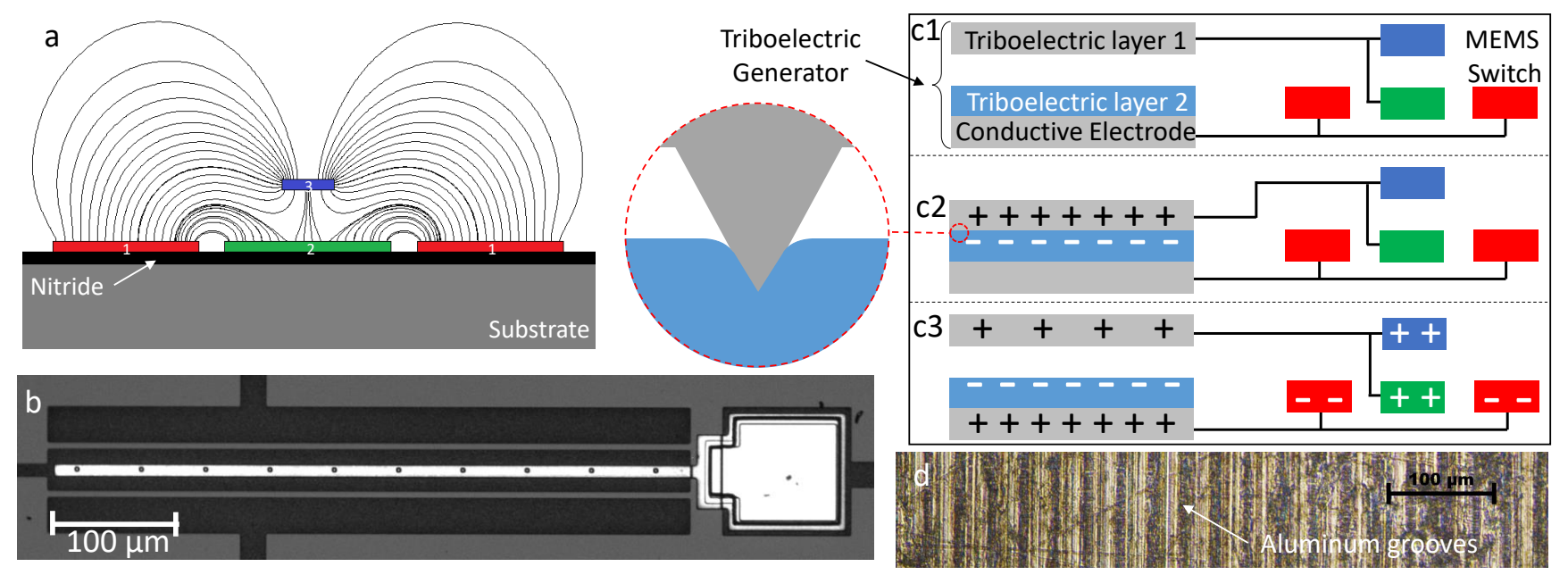

Figure 1. a) Electrostatic levitation electrode configuration with electric field lines. Side electrodes (1) are supplied with a large voltage, the center electrode (2) is given a small bias, and the beam is grounded. The electrodes are isolated from the substrate with a $0.5 \mu \mathrm{m}$ thick silicon nitride layer. b) Optical image of a fabricated switch. c) Schematic of the triboelectric generator (left) when connected to the MEMS switch (right) showing the working principle and charge migration. d) Top down optical image of the grooves on the bottom face of the aluminum triboelectric layer. As a force is applied to the generator, the grooves dig into the surface of the PDMS increasing contact area and microscopic sliding that greatly increases the voltage generated.

MEMS actuators because the high voltage output would immediately cause pull-in, which would create stiction between the MEMS beam and electrode, and discharge the generator. However, levitation-based MEMS avoid pull-in between the beam and side electrodes and draw no current, which allows the generator to remain completely open circuit and maintain a high voltage. This shows there is a synergy between these two mechanisms where the advantages of one addresses the limitations of the other, which could lead to the creation of a tremendous number of MEMS devices.

The contribution of this study is the innovative coupling of a MEMS electrostatic levitation switch with a triboelectric generator (Figure 2). We demonstrate that the triboelectric generator can drive a MEMS cantilever using electrostatic levitation, and furthermore, it can toggle the switch from closed to open. The switch opens when the applied force on the generator goes beyond a threshold. This result is not only promising for normallyclosed MEMS switches, but also opens up possibilities for MEMS applications by demonstrating the compatibility of triboelectricity and electrostatic levitation. It provides a realistic avenue for taking advantage of the high voltage output of triboelectric generators and a large, stable travel range with electrostatic levitation while eliminating the disadvantages each has independently. This principle can be employed in innumerable electrostatic devices to operate directly from mechanical vibration or shock for safety switches such as those in air bag deployment devices, safety helmets that can detect shock, as well as earthquake detection systems that need to trigger an immediate response to significant seismic activity.

The system consists of an open circuit triboelectric generator connected to an electrostatic levitation MEMS switch (Figure 2) with an electrode configuration shown in Figure 1 (a). The center electrode is given a small bias voltage to initiate pull-in and bring the center electrode and beam into contact with each other, which acts as a closed switch. The side electrodes are supplied with a large voltage to create levitation and pull the beam upward. When the side voltage passes a threshold value, the beam releases from its pulled in position and settles to a stable equilibrium position above the center electrode. This releasing mechanism was experimentally demonstrated by the authors in a previous study ${ }^{13}$ and a relationship between bias voltage and threshold release voltage was mapped.

The triboelectric generator is pictured in Figure 1 (c). It consists of an aluminum (top) and PDMS (bottom) triboelectric layer. Because the aluminum layer is conductive, it acts as its own electrode and is connected directly to the beam. The PDMS triboelectric layer is an insulator, so a conductive electrode is attached to its back to create an electrical connection to the side electrodes. When a force brings the two triboelectric materials into contact with each other, electrons transfer from the aluminum to the PDMS leaving the aluminum positively charged and the PDMS negatively charged. Once the layers are released, the positive charge spreads out to the beam and center electrode from the aluminum. Because the PDMS is an insulator, the gained electrons cannot easily move to the side electrodes. However, through electrostatic induction, the electrons in the back electrode are pushed over to the side electrodes giving it a negative charge. This produces a voltage potential between the center and side electrodes, which creates electrostatic levitation.

The amount of voltage produced on the actuating elec- 

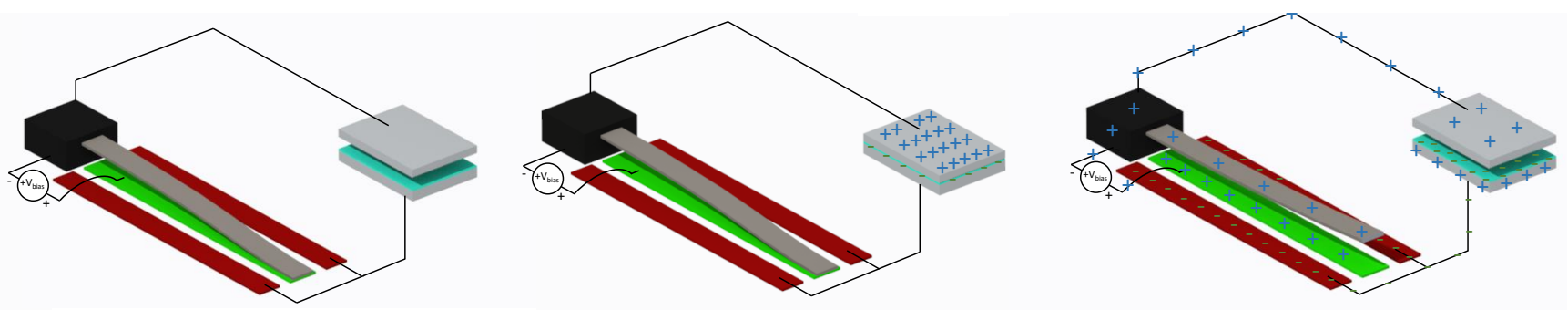

Figure 2. Schematic showing the generator and cantilever during each stage.

trodes is related to the amount of contact pressure between triboelectric layers with more pressure producing more voltage. This is a result of an increase in contact area between triboelectric layers with higher pressure, and microscopic sliding that occurs between surfaces, which increases triboelectric charges ${ }^{21}$. The increase in contact area is a result of the grooves machined into the bottom face of the aluminum digging into the surface of the PDMS as pictured in Figure 1 (c).

A schematic of the total sensor/switch system is pictured in Figure 2. A voltage source is placed between the beam and center electrode to apply the bias voltage. This voltage induces the attractive electrostatic force and initiates pull-in. The triboelectric layers are then pressed together and released, generating a large voltage between center and side electrodes. If the contact pressure is large enough, the levitation force overcomes the attractive electrostatic force from the bias voltage and the beam releases (switch opens) (Figure 2).

The MEMS switch is built using the PolyMUMPs standard fabrication procedure ${ }^{22}$. The generator was built as outlined in a previous study ${ }^{20}$ and has an area of approximately $20 \mathrm{~cm}^{2}$. The switch is mounted on a vibration isolator at atmospheric pressure and the beam tip displacement is measured with a Polytec OFV-534 portable laser vibrometer. The experiment is controlled in MATLAB with a National Instruments USB 6251 Data Acquisition system taking displacement data from the laser and measuring bias voltage. A B\& K Precision 9110 power supply applies the bias voltage to the center electrode of the switch. An MTS 858 Bionix Mini Test System applies pressure to the generator and measures the applied force. The generator voltage is measured with a Keithley 6514 electrometer. A schematic of the experimental setup is shown in Figure 3.

The experimental data demonstrating the sensorswitch concept is shown in Figure 4. First, the bias voltage is ramped up to $5.6 \mathrm{~V}$ to initiate pull-in. As the bias voltage increases, the beam moves downward before suddenly pulling in and sticking to the substrate at around $3 \mathrm{~s}$. The bias voltage is reduced to $2 \mathrm{~V}$ to lower the threshold voltage needed to release the beam. Next, the MTS applies a $1 \mathrm{~Hz}$ half-sine force on the generator with a peak of approximately $3.6 \mathrm{kN}$ near $15 \mathrm{~s}$. During contact, the generator outputs about $-50 \mathrm{~V}$, but when the layers are released the voltage instantly spikes to $150 \mathrm{~V}$ and be-

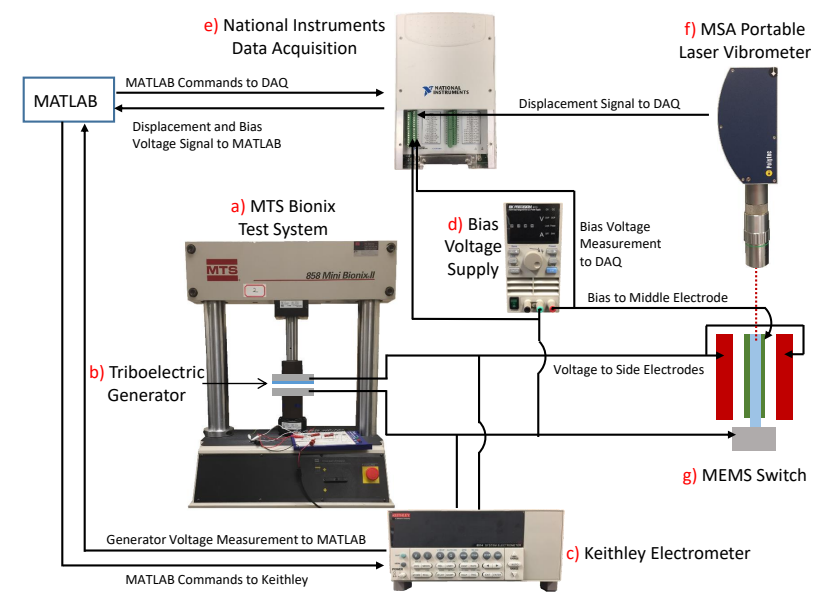

Figure 3. Set up of the experiment.

gins to decay. At the same time, the beam jumps up to a height of $16 \mu \mathrm{m}$ and begins to slowly fall back down as the generator discharges. A previous study demonstrated that higher frequencies for the input force will yield more output voltage $\mathrm{e}^{23}$.

The results show the compatibility of the two systems. The switch opens only if the applied pressure goes beyond a limit. It demonstrates that a triboelectric generator can be used as the toggling mechanism for the electrostatic levitation MEMS switch previously proposed by the authors ${ }^{13}$. This creates the possibility for normallyclosed MEMS switches that can be toggled open by harvesting ambient force or vibration energy. An example of how this could be used would be for a safety sensor and switch that detects when an object has received an impact, converts some of the impact energy to electrical energy, and uses that energy to toggle open a switch and cut power to a circuit if the impact is above a threshold level. This could have numerous benefits for monitoring and safety applications.

The switch can be modeled as an Euler-Bernoulli beam that is governed by the partial differential equation in Equation (1),

$$
\rho A \frac{\partial^{2} \hat{w}}{\partial \hat{t}^{2}}+\hat{c} \frac{\partial \hat{w}}{\partial \hat{t}}+E I \frac{\partial^{4} \hat{w}}{\partial \hat{x}^{4}}=\hat{f}_{e}(\hat{w}, \vec{V})
$$

where $\hat{w}$ is the transverse deflection, $\rho$ is the density $\left(2330 \mathrm{~kg} / \mathrm{m}^{3}\right), \mathrm{A}$ is the area of the cross-section $\left(20 \mu \mathrm{m}^{2}\right)$, 

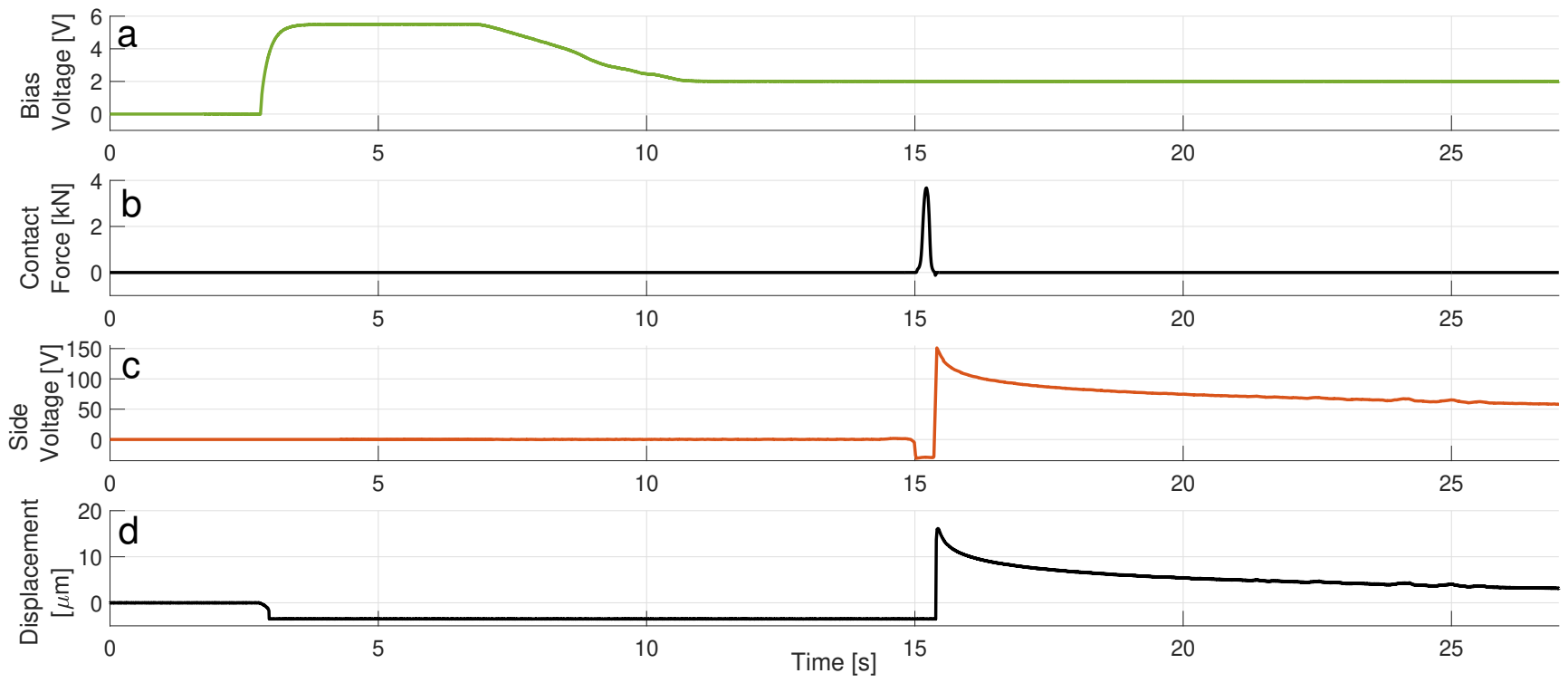

Figure 4. Experimental data showing a) bias voltage, b) contact force, c) generator voltage, and d) beam tip displacement.

$\hat{c}$ is the damping coefficient, $\mathrm{E}$ is the elastic modulus $(160 \mathrm{GPa}), \mathrm{I}$ is the second moment of the area $\left(6.67 \mu \mathrm{m}^{4}\right)$, and $\hat{f}_{e}(\hat{w}, \vec{V})$ is the electrostatic force that depends on the deflection and applied voltages.

Unlike conventional parallel-plate electrodes that have a closed-form expression for the electrostatic force, here the orientation of electrodes is unusual, making the derivation of the force complicated. Therefore, the force is calculated from the derivative of the electrostatic potential energy, which depends on the capacitance of the system. The capacitance is calculated with a $2 \mathrm{D}$ finite element simulation in COMSOL. Because there are three electrodes (taking the two side electrodes as a single electrode), there are capacitances between each pair of electrodes. The potential energy function becomes

$$
U=\frac{1}{2}\left[\begin{array}{lll}
V_{1} & V_{2} & V_{3}
\end{array}\right]\left[\begin{array}{lll}
c_{11} & c_{12} & c_{13} \\
c_{21} & c_{22} & c_{23} \\
c_{31} & c_{32} & c_{33}
\end{array}\right]\left[\begin{array}{l}
V_{1} \\
V_{2} \\
V_{3}
\end{array}\right]
$$

where $c_{i j}$ are the capacitances between each pair of electrodes and $V_{i}$ are the voltages on each electrode as defined in Figure 1 (a). Multiplying Equation (2) out, setting $V_{3}=0$ because the beam is assumed to be at the reference ground level, and taking the derivative with respect to $w$, yields the forcing term.

$$
f_{e}(w, \vec{V})=\frac{1}{2}\left(V_{1}^{2} \frac{\partial c_{11}}{\partial w}+2 V_{1} V_{2} \frac{\partial c_{12}}{\partial w}+V_{2}^{2} \frac{\partial c_{22}}{\partial w}\right)
$$

With the appropriate fitting functions for $\frac{c_{11}}{\partial w}, \frac{c_{12}}{\partial w}$, and $\frac{c_{22}}{\partial w}$, the static behavior of the switch can be predicted with excellent accuracy. Figure 5 shows the static tip deflection as a function of side voltages for $6 \mathrm{~V}$ bias when the side voltage is swept up to $200 \mathrm{~V}$. The model and experiment show very close agreement verifying the static
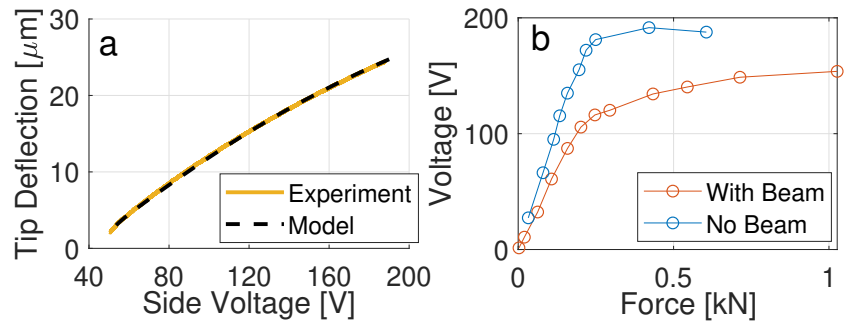

Figure 5. a) Static tip deflection as a function of side voltage for the model and experiment at $6 \mathrm{~V}$ bias. b) Open circuit voltage versus contact force for the triboelectric generator with and without the switch.

model and the derivation of electrostatic force. The beam is $500 \mu \mathrm{m}$ in length and $2 \mu \mathrm{m}$ thick. The beam tip is capable of reaching $25 \mu \mathrm{m}$ despite only being anchored $2 \mu \mathrm{m}$ above the center electrode. An in-depth static and dynamic analysis of the MEMS component was previously performed by the authors ${ }^{24}$.

While the switch can be modeled with high accuracy, the generator is much more difficult to model. However, the relationship between input force and output voltage can be characterized experimentally by pressing the triboelectric layers together at varying pressure levels and measuring the output voltage [Figure 5 (b)].

The generator is capable of outputting almost $200 \mathrm{~V}$ when disconnected from the beam. There is a linear relationship between force and voltage until approximately $200 \mathrm{~N}$, after which the voltage begins to saturate. When the switch is connected, the output voltage saturates around $150 \mathrm{~V}$. This difference is a result of the resistance between the center and side electrodes through the silicon nitride pictured in Figure 1 (a). In this case, the volt- 
age decays in a similar manner as a discharging capacitor through a resistor. When the switch is disconnected, the voltage stays relatively constant. The discharge rate can be reduced by increasing the resistance between the center and side electrodes by increasing the thickness of silicon nitride or by using an insulator with a higher resistivity. To mitigate this issue, the bias voltage was turned down to $2 \mathrm{~V}$ so the generator was still capable of releasing the beam despite the decrease in voltage.

The switch model and generator characterization give a complete picture of the sensor/switch system. The experimental mapping of bias voltage vs release voltage previously performed by the authors ${ }^{13}$ can be used with the force-voltage relationship of the generator to control the threshold force required to toggle the switch. The switch is scalable to smaller sizes. Smaller switch dimensions will actually boost the strength of the levitation force at its peak and could further reduce the threshold release voltage of the switch ${ }^{25}$. The model helps with designing MEMS where the motion needs to be predicted. The sensor switch system requires no external power to open the switch. It has no running cost and requires no computational overhead. Applications that needs to detect a force and trigger an action would benefit from this system. Some examples include air bag systems in motor vehicles that detect when a collision has occurred and deploy the air bag, safety helmets that provide local information on the severity of the impact, or earthquake detection systems that trigger in response to significant seismic activity. This study opens the gateway for completely autonomous MEMS sensors and switches based on electrostatic levitation and triboelectricity.

Authors acknowledge the National Science Foundation for supporting this study through grants CMMI \# 1919608 and ECCS \# 1608692.

\section{REFERENCES}

${ }^{1}$ M. I. Younis, Mems Linear and Nonlinear Statics and Dynamics (Springer, New York, 2011).

${ }^{2}$ W.-M. Zhang, G. Meng, and D. Chen, "Stability, nonlinearity and reliability of electrostatically actuated mems devices," Sensors 7, 760-796 (2007).

${ }^{3}$ K. B. Lee and Y. H. Cho, "Laterally driven electrostatic repulsive-force microactuators using asymmetric field distribution," Journal of Microelectromechanical Systems 10, 128-136 (2001).

${ }^{4}$ Q. Chen, Y.-C. Lai, J. Chae, and Y. Do, "Anti-phase synchronization in microelectromechanical systems and effect of impulsive perturbations," Physical Review B 87, 144304 (6pp.) (2013).

${ }^{5}$ Y. Linzon, B. Ilic, S. Lulinsky, and S. Krylov, "Efficient parametric excitation of silicon-on-insulator microcantilever beams by fringing electrostatic fields," Journal of Applied Physics 113 (2013), 10.1063/1.4802680.

${ }^{6}$ R. N. Miles, "A Compliant Capacitive Sensor for Acoustics: Avoiding Electrostatic Forces at High Bias Voltages," IEEE Sensors 18, $5691-5698$ (2018).
${ }^{7}$ S. He and R. Ben Mrad, "Large-stroke microelectrostatic actuators for vertical translation of micromirrors used in adaptive optics," IEEE Transactions on Industrial Electronics 52, 974-983 (2005).

${ }^{8} \mathrm{~S}$. He and R. Ben Mrad, "Design, modeling, and demonstration of a MEMS repulsive-force out-of-plane electrostatic micro actuator," Journal of Microelectromechanical Systems 17, 532-547 (2008).

${ }^{9}$ C. Fan and S. He, "A Two-Row Interdigitating-Finger RepulsiveTorque Electrostatic Actuator and Its Application to Micromirror Vector Display," Journal of Microelectromechanical Systems 24, 2049-2061 (2015).

${ }^{10}$ M. Pallay, M. Daeichin, and S. Towfighian, "Dynamic Behavior of an Electrostatic MEMS Resonator with Repulsive Actuation," Nonlinear Dynamics 89, 1525-1538 (2017).

${ }^{11}$ M. Pallay and S. Towfighian, "A parametric electrostatic resonator using repulsive force," Sensors and Actuators A: Physical 277, 134 - 141 (2018).

${ }^{12}$ M. Ozdogan, M. Daeichin, A. Ramini, and S. Towfighian, "Parametric Resonance of a Repulsive Force MEMS Electrostatic Mirror," Sensors and Actuators A Physical, 265, 20-31 (2017).

${ }^{13}$ M. Pallay and S. Towfighian, "A reliable MEMS switch using electrostatic levitation," Applied Physics Letters 113, 213102 (2018).

${ }^{14}$ W. Du, X. Han, L. Lin, M. Chen, X. Li, C. Pan, and Z. L. Wang, "A three dimensional multi-layered sliding triboelectric nanogenerator," Advanced Energy Materials 4 (2014).

${ }^{15}$ Y. Xie, S. Wang, S. Niu, L. Lin, Q. Jing, J. Yang, Z. Wu, and Z. L. Wang, "Grating-structured freestanding triboelectric-layer nanogenerator for harvesting mechanical energy at $85 \%$ total conversion efficiency," Advanced Materials 26, 6599-6607 (2014).

${ }^{16}$ G. Zhu, C. Pan, W. Guo, C.-Y. Chen, Y. Zhou, R. Yu, and Z. L. Wang, "Triboelectric-generator-driven pulse electrodeposition for micropatterning," Nano letters 12, 4960-4965 (2012).

${ }^{17}$ S. Niu, Y. Liu, S. Wang, L. Lin, Y. S. Zhou, Y. Hu, and Z. L. Wang, "Theory of sliding-mode triboelectric nanogenerators," Advanced Materials 25, 6184-6193 (2013).

${ }^{18}$ G. Zhu, J. Chen, T. Zhang, Q. Jing, and Z. L. Wang, "Radialarrayed rotary electrification for high performance triboelectric generator," Nature communications 5 (2014).

${ }^{19}$ L. Lin, S. Wang, S. Niu, C. Liu, Y. Xie, and Z. L. Wang, "Noncontact free-rotating disk triboelectric nanogenerator as a sustainable energy harvester and self-powered mechanical sensor," ACS applied materials \& interfaces 6, 3031-3038 (2014).

${ }^{20}$ A. Ibrahim, A. Ramini, and S. Towfighian, "Experimental and theoretical investigation of an impact vibration harvester with triboelectric transduction," Journal of Sound and Vibration 416, 111-124 (2018).

${ }^{21}$ C. Jin, D. S. Kia, M. Jones, and S. Towfighian, "On the contact behavior of micro-/nano-structured interface used in verticalcontact-mode triboelectric nanogenerators," Nano Energy 27, 68-77 (2016).

${ }^{22}$ A. Cowen, B. Hardy, R. Mahadevan, and S. Wilcenski, "PolyMUMPs Design Handbook a MUMPs@ process," (2011).

${ }^{23}$ S. Wang, L. Lin, and Z. L. Wang, "Nanoscale triboelectric-effectenabled energy conversion for sustainably powering portable electronics," Nano letters 12, 6339-6346 (2012).

${ }^{24}$ M. Pallay, R. N. Miles, and S. Towfighian, "Merging parallelplate and levitation actuators to enable linearity and tunability in electrostatic mems," Journal of Applied Physics 126, 014501 (2019).

${ }^{25}$ S. Towfighian, S. He, and R. Ben Mrad, "A low voltage electrostatic micro actuator for large out-of-plane displacement," in ASME 2014 International Design Engineering Technical Conferences and Computers and Information in Engineering Conference (American Society of Mechanical Engineers Digital Collection, 2014) p. 7 pages. 